\title{
Chirality from Interfacial Spin-Orbit Coupling Effects in Magnetic Bilayers
}

\author{
Kyoung-Whan Kim ${ }^{1,2}$, Hyun-Woo Lee 2 * Kyung-Jin Lee Le $^{3,4}$ and M. D. Stiles ${ }^{5}$ \\ ${ }^{1}$ Basic Science Research Institute, Pohang University of Science and Technology, Pohang, 790-784, Korea \\ ${ }^{2}$ Department of Physics, Pohang University of Science and Technology, Pohang, 790-784, Korea \\ ${ }^{3}$ Department of Materials Science and Engineering, Korea University, Seoul, 136-701, Korea \\ ${ }^{4}$ KU-KIST Graduate School of Converging Science and Technology, Korea University, Seoul 136-713, Korea \\ ${ }^{5}$ Center for Nanoscale Science and Technology, National Institute of \\ Standards and Technology, Gaithersburg, Maryland 20899-6202, USA
}

(Dated: March 2, 2022)

\begin{abstract}
As nanomagnetic devices scale to smaller sizes, spin-orbit coupling due to the broken structural inversion symmetry at interfaces becomes increasingly important. Here we study interfacial spinorbit coupling effects in magnetic bilayers using a simple Rashba model. The spin-orbit coupling introduces chirality into the behavior of the electrons and through them into the energetics of the magnetization. In the derived form of the magnetization dynamics, all of the contributions that are linear in the spin-orbit coupling follow from this chirality, considerably simplifying the analysis. For these systems, an important consequence is a correlation between the Dzyaloshinskii-Moriya interaction and the spin-orbit torque. We use this correlation to analyze recent experiments.
\end{abstract}

Magnetic bilayers that consist of an atomically thin ferromagnetic layer (such as Co) in contact with a nonmagnetic layer (such as $\mathrm{Pt}$ ) with strong spin-orbit coupling have emerged as prototypical systems that exhibit very strong spin-orbit coupling effects. Strong spin-orbit coupling can enhance the efficiency of the electrical control of magnetization. A series of recent experiments [1[4] on magnetic bilayers report dramatic effects such as anomalously fast current-driven magnetic domain wall motion [2] and reversible switching of single ferromagnetic layers by in-plane currents [3, 4]. Strong spin-orbit coupling can introduce chirality into the magnetic ground state [5, 6]. This chirality is predicted [7] to boost the electrical control of magnetic degrees of freedom even further as has been confirmed in two experiments [8, 9].

Interfaces lack structural inversion symmetry, allowing interfacial spin-orbit coupling to play an expanded role. In magnetic bilayers, it generates various effects including the Dzyaloshinskii-Moriya (DM) interaction 10 12 and the spin-orbit torque 13 18]. Here, we examine a simple Rashba model of the interface region. We compute the equation of motion for a magnetization texture $\hat{\mathbf{m}}(\mathbf{r})$ by integrating out the electron degrees of freedom. We report two main findings. The first is the correlation between the DM interaction and the spin-orbit torques. Spin-orbit torques arise from interfacial spin-orbit coupling but also from the bulk spin Hall effect, and the importance of each contribution is hotly debated [3, 19 22]. The correlation we find opens a way to quantify the contribution from interfacial spin-orbit coupling by measuring the DM interaction, allowing one to disentangle the two contributions.

The second finding is that all linear effects of the interfacial spin-orbit coupling, including the DM interaction and the spin-orbit torque, can be captured through a simple mathematical construct, which we call a chiral derivative. The chiral derivative also shows in the equa- tion of motion how each contribution that is linear in the spin-orbit coupling corresponds to a contribution that is present even in the absence of spin-orbit coupling. This correspondence provides a simple way to quantitatively predict and understand a wide variety of interfacial spinorbit coupling effects allowed by symmetry [18. In the last part of the Letter, we discuss briefly the extension to realistic situations, which go beyond the simple Rashba model.

Our analysis begins with the two-dimensional (2D) Rashba Hamiltonian

$$
\begin{aligned}
\mathcal{H} & =\mathcal{H}_{\mathrm{kin}}+\mathcal{H}_{\mathrm{R}}+\mathcal{H}_{\mathrm{exc}}+\mathcal{H}_{\mathrm{imp}} \\
& =\frac{\mathbf{p}^{2}}{2 m_{e}}+\frac{\alpha_{\mathrm{R}}}{\hbar} \boldsymbol{\sigma} \cdot(\mathbf{p} \times \hat{\mathbf{z}})+J \boldsymbol{\sigma} \cdot \hat{\mathbf{m}}+\mathcal{H}_{\mathrm{imp}}
\end{aligned}
$$

where $\mathbf{p}$ is the $2 \mathrm{D}$ electron momentum in the $x y$ plane, the vector $\boldsymbol{\sigma}$ of the Pauli matrices represents the electron spin, and $|\hat{\mathbf{m}}(\mathbf{r})|=1$. $\mathcal{H}$ is a minimal model 13 -18] for electronic properties of the interface region between the ferromagnetic and nonmagnetic layers in magnetic bilayers, and captures the broken symmetries; $\mathcal{H}_{\text {exc }}$ breaks the time-reversal symmetry, and $\mathcal{H}_{\mathrm{R}}$ breaks the structural inversion symmetry. The last term $\mathcal{H}_{\text {imp }}$ describes the scattering by both spin-independent and quenched spin-dependent impurities. The latter part of $\mathcal{H}_{\text {imp }}$ contributes to the Gilbert damping and the nonadiabatic spin torque [23, 24].

Here, we focus on effects of $\mathcal{H}_{R}$ on the equation of motion for the magnetization up to order $\alpha_{R}$. These effects include the DM interaction and the spin-orbit torque. We neglect effects of order $\alpha_{\mathrm{R}}^{2}$ such as interfaceinduced magnetic anisotropy, contributions to Gilbert damping [25, 26], and to the nonadiabaticity parameter [27]. We introduce the unitary transformation [28, 29]

$$
\mathcal{U}=\exp \left[-i k_{\mathrm{R}} \boldsymbol{\sigma} \cdot(\mathbf{r} \times \hat{\mathbf{z}}) / 2\right],
$$


where

$$
k_{\mathrm{R}}=\frac{2 \alpha_{\mathrm{R}} m_{e}}{\hbar^{2}}
$$

and $\mathbf{r}=(x, y) . \mathcal{U}$ rotates the electron spin around the $\hat{\mathbf{r}} \times \hat{\mathbf{z}}$ direction by the angle $k_{\mathrm{R}} r$, where $r=|\mathbf{r}|$. We also introduce the $\mathbf{r}$-dependent $3 \times 3$ matrix $\mathcal{R}$, which achieves the same rotation of a classical vector such as $\hat{\mathbf{m}}$. Upon the unitary transformation, one finds (Supplementary Material [30])

$$
\mathcal{U}^{\dagger} \mathcal{H} \mathcal{U}=\mathcal{H}_{\text {kin }}+J \boldsymbol{\sigma} \cdot \hat{\mathbf{m}}^{\prime}+\mathcal{H}_{\mathrm{imp}}^{\prime}+\mathcal{O}\left(\alpha_{\mathrm{R}}^{2}\right),
$$

where

$$
\hat{\mathbf{m}}^{\prime}=\mathcal{R}^{-1} \hat{\mathbf{m}}
$$

and $\mathcal{H}_{\text {imp }}^{\prime}=\mathcal{U}^{\dagger} \mathcal{H}_{\text {imp }} \mathcal{U}$. We ignore the last term in Eq. (4) as higher order. $\mathcal{H}_{\mathrm{imp}}^{\prime}$ is not identical to $\mathcal{H}_{\text {imp }}$ but they share the same impurity expectation values up to $\mathcal{O}\left(\alpha_{\mathrm{R}}\right)$, which implies that $\mathcal{H}_{\mathrm{R}}$ has no effect to linear order on the Gilbert damping coefficient or the nonadiabaticity coefficient [23, 24]. Thus up to $\mathcal{O}\left(\alpha_{\mathrm{R}}\right), \mathcal{H}_{\mathrm{imp}}^{\prime}$ may be identified with $\mathcal{H}_{\text {imp. }}$. Then the unitary transformation from $\mathcal{H}$ to $\mathcal{U}^{\dagger} \mathcal{H U}$ has eliminated $\mathcal{H}_{\mathrm{R}}$ at the expense of replacing $\hat{\mathbf{m}}$ by $\hat{\mathbf{m}}^{\prime}$.

With this replacement, we compute the energy of the filled Fermi sea as a function of $\hat{\mathbf{m}}$. Without $\mathcal{H}_{\mathrm{R}}$, the energy can depend on $\hat{\mathbf{m}}$ only through spatial derivatives $\partial_{u} \hat{\mathbf{m}}(u=x, y)$ since the energy cannot depend on the direction of $\hat{\mathbf{m}}$ when $\hat{\mathbf{m}}$ is homogeneous. For $\hat{\mathbf{m}}$ smoothly varying over length scales longer than the Fermi wavelength, the energy density $\varepsilon$ may be expressed as the micromagnetic exchange interaction density $\varepsilon=$ $A\left(\partial_{x} \hat{\mathbf{m}} \cdot \partial_{x} \hat{\mathbf{m}}+\partial_{y} \hat{\mathbf{m}} \cdot \partial_{y} \hat{\mathbf{m}}\right)$, where $A$ is the interfacial exchange stiffness coefficient. Equation (4) implies that in the presence of $\mathcal{H}_{\mathrm{R}}, \varepsilon$ can be obtained simply by replacing $\partial_{u} \hat{\mathbf{m}}$ with $\partial_{u} \hat{\mathbf{m}}^{\prime} ; \varepsilon=A\left(\partial_{x} \hat{\mathbf{m}}^{\prime} \cdot \partial_{x} \hat{\mathbf{m}}^{\prime}+\partial_{y} \hat{\mathbf{m}}^{\prime} \cdot \partial_{y} \hat{\mathbf{m}}^{\prime}\right)$. One then uses the relation (Supplementary Information)

$$
\partial_{u} \hat{\mathbf{m}}^{\prime}=\partial_{u}\left(\mathcal{R}^{-1} \hat{\mathbf{m}}\right)=\mathcal{R}^{-1} \tilde{\partial}_{u} \hat{\mathbf{m}},
$$

where the chiral derivative $\tilde{\partial}_{u}$ is defined by

$$
\tilde{\partial}_{u} \hat{\mathbf{m}}=\partial_{u} \hat{\mathbf{m}}+k_{\mathrm{R}}(\hat{\mathbf{z}} \times \hat{\mathbf{u}}) \times \hat{\mathbf{m}} .
$$

Here $\hat{\mathbf{u}}$ is the unit vector along the direction $u$. The second term in Eq. (7) arises from the derivative operator acting on the $\mathbf{r}$-dependent $\mathcal{R}^{-1}$. $\varepsilon$ in the presence of the interfacial spin-orbit coupling then becomes

$$
\begin{aligned}
\varepsilon= & A\left(\partial_{x} \hat{\mathbf{m}} \cdot \partial_{x} \hat{\mathbf{m}}+\partial_{y} \hat{\mathbf{m}} \cdot \partial_{y} \hat{\mathbf{m}}\right) \\
& +D\left[\hat{\mathbf{y}} \cdot\left(\hat{\mathbf{m}} \times \partial_{x} \hat{\mathbf{m}}\right)-\hat{\mathbf{x}} \cdot\left(\hat{\mathbf{m}} \times \partial_{y} \hat{\mathbf{m}}\right)\right]+\mathcal{O}\left(\alpha_{\mathrm{R}}^{2}\right),
\end{aligned}
$$

with

$$
D=2 k_{\mathrm{R}} A \text {. }
$$

Note that the second term in Eq. (8) is nothing but the interfacial DM interaction responsible for chiral magnetic order addressed recently [7 9]. A few remarks are in order. First, this derivation shows that the DM interaction is intimately related to the usual micromagnetic exchange interaction that exists even in the absence of interfacial spin-orbit coupling. This is the first example of the one-to-one correspondence and illustrates how the interfacial spin-orbit coupling generates a term in linear order from each term present in the absence of the spinorbit coupling. Second, this mechanism for the DM interaction in an itinerant ferromagnet is similar to that of the Ruderman-Kittel-Kasuya-Yosida interaction in nonmagnetic systems acquiring the DM-like character [31, 32] when conduction electrons are subject to interfacial spinorbit coupling.

Next, we demonstrate the correlation between the DM interaction and the spin-orbit torque. Although the spinorbit torque has already been derived from Eq. (10) in previous studies [13 18], we present below a derivation of the spin-orbit torque that shows the relationship between it and the DM interaction. Without $\mathcal{H}_{\mathrm{R}}$, it is well known [33] that the total spin torque $\mathbf{T}_{\text {st }}$ induced by an in-plane current density $\mathbf{j}$ consists of the following two components,

$$
\mathbf{T}_{\mathrm{st}}=v_{\mathrm{s}}(\hat{\mathbf{j}} \cdot \boldsymbol{\nabla}) \hat{\mathbf{m}}-\beta v_{\mathrm{s}} \hat{\mathbf{m}} \times(\hat{\mathbf{j}} \cdot \boldsymbol{\nabla}) \hat{\mathbf{m}},
$$

where the first and the second components are the adiabatic [34] and nonadiabatic [35, 36] spin toques, respectively. Here $\hat{\mathbf{j}}=\mathbf{j} / j, j=|\mathbf{j}|, \beta$ is the nonadiabaticity parameter [35, 36], and the spin velocity $v_{\mathrm{s}}=$ $P j g \mu_{\mathrm{B}} /\left(2 e M_{\mathrm{s}}\right)$, where $P$ is the polarization of the current, $g$ is the Landé $g$ factor, $\mu_{\mathrm{B}}$ is the Bohr magneton, $M_{\mathrm{S}}$ is the saturation magnetization, and $-e(<0)$ is the electron charge. In the presence of $\mathcal{H}_{\mathrm{R}}$, Eqs. (4) and (6) imply that $\mathbf{T}_{\mathrm{st}}$ changes to

$$
\mathbf{T}_{\mathrm{st}}=v_{\mathrm{s}}(\hat{\mathbf{j}} \cdot \tilde{\nabla}) \hat{\mathbf{m}}-\beta v_{\mathrm{s}} \hat{\mathbf{m}} \times(\hat{\mathbf{j}} \cdot \tilde{\nabla}) \hat{\mathbf{m}},
$$

where $\tilde{\nabla}=\left(\tilde{\partial}_{x}, \tilde{\partial}_{y}\right)$. One then obtains from Eq. (7)

$$
\begin{aligned}
\mathbf{T}_{\mathrm{st}} & =v_{\mathrm{s}}(\hat{\mathbf{j}} \cdot \nabla) \hat{\mathbf{m}}-\beta v_{\mathrm{s}} \hat{\mathbf{m}} \times(\hat{\mathbf{j}} \cdot \nabla) \hat{\mathbf{m}} \\
& +\tau_{\mathrm{f}} v_{\mathrm{s}} \hat{\mathbf{m}} \times(\hat{\mathbf{j}} \times \hat{\mathbf{z}})-\tau_{\mathrm{d}} v_{\mathrm{s}} \hat{\mathbf{m}} \times[\hat{\mathbf{m}} \times(\hat{\mathbf{j}} \times \hat{\mathbf{z}})] .
\end{aligned}
$$

The two terms in the second line are the two components of the spin-orbit torque. The first (second) component in the second line is called the fieldlike (dampinglike) spinorbit torque and arises from the adiabatic (nonadiabatic) torque in the first line. This is the second example of the one-to-one correspondence. The chiral derivative fixes the coefficients of the two spin-orbit torque components to

$$
\tau_{\mathrm{f}}=k_{\mathrm{R}}, \tau_{\mathrm{d}}=\beta k_{\mathrm{R}} .
$$

When combined with Eq. (9), one finds

$$
\tau_{\mathrm{f}}=D / 2 A, \tau_{\mathrm{d}}=\beta D / 2 A .
$$


This correlation between the DM coefficient $D$ and the spin-orbit torque coefficients $\tau_{\mathrm{f}}$ and $\tau_{\mathrm{d}}$ is a key result of this work.

A recent experiment [8] examined current-driven domain wall motion in the systems $\mathrm{Pt} / \mathrm{CoFe} / \mathrm{MgO}$ and $\mathrm{Ta} / \mathrm{CoFe} / \mathrm{MgO}$ and concluded that domain wall motion against (along) the electron flow in the former (latter) system is due to the product $D \tau_{\mathrm{d}} P$ being positive (negative). According to Eqs. (9) and (13), $D \tau_{\mathrm{d}} P=2 \beta P A k_{\mathrm{R}}^{2}$ should be of the same sign as $\beta P$ regardless of $k_{\mathrm{R}}$ since $A$ is positive by definition. Thus explaining the experimental results for $\mathrm{Ta} / \mathrm{CoFe} / \mathrm{MgO}$ within the interfacial spin-orbit coupling theory requires $\beta P$ to be negative. Whereas $\beta P$ can be negative, in most models and parameter ranges it is positive. We tentatively conclude that $\tau_{\mathrm{d}}$ in $\mathrm{Ta} / \mathrm{CoFe} / \mathrm{MgO}[8]$ has a different origin, the spin Hall effect being a plausible mechanism as argued in Ref. [8]. For $\mathrm{Pt} / \mathrm{CoFe} / \mathrm{MgO}$, on the other hand, the reported sign is consistent with the sign determined from Eqs. (9) and (13) if $\beta P>0$. The Pt-based structure in Ref. [9] also gave the same sign as Ref. [8]. To investigate the origin of the spin-orbit torque in $\mathrm{Pt} / \mathrm{CoFe} / \mathrm{MgO}$, we attempt a semiquantitative analysis. For the suggested values $D=0.5 \mathrm{~mJ} / \mathrm{m}^{2}, A=10^{-11} \mathrm{~J} / \mathrm{m}$ in Ref. [8], Eq. (9) predicts $k_{\mathrm{R}}=2.5 \times 10^{8} \mathrm{~m}^{-1}$. For $P=0.5$, $\beta=0.4, M_{\mathrm{s}}=3 \times 10^{5} \mathrm{Am}^{-1}$, which are again from Ref. [8], Eq. (13) predicts the effective transverse field $-\left(\tau_{\mathrm{f}} v_{\mathrm{s}} / \gamma\right) \hat{\mathbf{j}} \times \hat{\mathbf{z}}$ of the fieldlike spin-orbit torque and the effective longitudinal field $\left(\tau_{\mathrm{d}} v_{\mathrm{s}} / \gamma\right)(\hat{\mathbf{m}} \times(\hat{\mathbf{m}} \times \hat{\mathbf{z}}))$ of the dampinglike spin-orbit torque to have the magnitudes $1.3 \mathrm{mT}$ and $0.52 \mathrm{mT}$, respectively, for $j=10^{11} \mathrm{~A} / \mathrm{m}^{2}$. Here $\gamma$ is the gyromagnetic ratio. The former value is in reasonable agreement with the measured value $2 \mathrm{mT}$ considering uncertainty in the parameter values quoted above, whereas the latter value is about an order of magnitude smaller than the measured value $5 \mathrm{mT}$ in Ref. [8]. We thus conclude that the fieldlike spin-orbit torque of $\mathrm{Pt} / \mathrm{CoFe} / \mathrm{MgO}$ in Ref. [8] is probably due to the interfacial spin-orbit coupling whereas the dampinglike spinorbit torque is probably due to a different mechanism such as the bulk spin Hall effect. For the fieldlike spinorbit torque of $\mathrm{Pt} / \mathrm{CoFe} / \mathrm{MgO}$, the relative sign of $\tau_{\mathrm{f}}$ with respect to $D$ is also consistent with the prediction of the interfacial spin-orbit coupling if $P$ is positive.

These two examples illustrate the idea that all linear effects of the interfacial spin-orbit coupling can be captured through the chiral derivative $\tilde{\partial}_{u} \hat{\mathbf{m}}$. To gain insight into its physical meaning, it is illustrative to take $u=x$ and examine the solution of $\tilde{\partial}_{x} \hat{\mathbf{m}}=0$, which forms a lefthanded (for $k_{\mathrm{R}}>0$ ) cycloidal spiral (Fig. 1), where $\hat{\mathbf{m}}$ precesses around the $-(\hat{\mathbf{z}} \times \hat{\mathbf{x}})$ axis $[-(\hat{\mathbf{z}} \times \hat{\mathbf{y}})$ axis if $u=y]$ as $x$ increases with the precession rate $d \theta / d x=k_{\mathrm{R}}$. This chiral precession gives the name, chiral derivative. Note that this precession is identical to the conduction electron spin precession caused by $\mathcal{H}_{\mathrm{R}}$ in nonmagnetic systems [37]. Moreover when $\tilde{\partial}_{x} \hat{\mathbf{m}}=0, \mathcal{H}_{\text {exc }}$ also causes

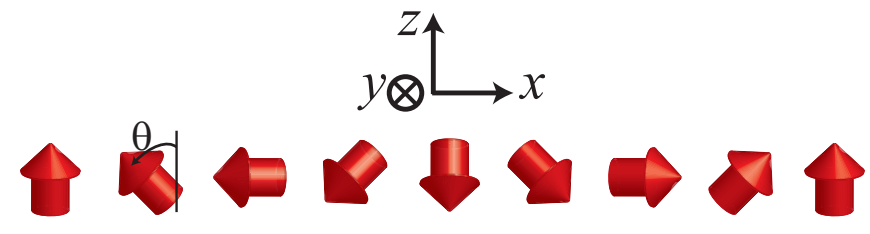

FIG. 1: (color online) Chiral precession of magnetization $\hat{\mathbf{m}}$. Chiral precession profile of $\hat{\mathbf{m}}$ with $\tilde{\partial}_{x} \hat{\mathbf{m}}=0$ forms a lefthanded (for $k_{\mathrm{R}}>0$ ) cycloidal spiral. This profile is identical to the spin precession profile of conduction electrons moving in the $+x$ or $-x$ direction in nonmagnetic systems with $\mathcal{H}_{\mathrm{R}}$ [37].

the same conduction electron spin precession as $\mathcal{H}_{\mathrm{R}}$ does. Thus effects of $\mathcal{H}_{\mathrm{R}}$ and $\mathcal{H}_{\text {exc }}$ become harmonious and the one-dimensional "half" $p_{x}^{2} / 2 m_{e}-\left(\alpha_{\mathrm{R}} / \hbar\right) \sigma_{y} p_{x}+J \boldsymbol{\sigma} \cdot \hat{\mathbf{m}}$ of the 2D Hamiltonian (1) gets minimized when $\tilde{\partial}_{x} \hat{\mathbf{m}}=0$. Interestingly, the sum of the exchange energy and the DM interaction, namely $A \partial_{x} \hat{\mathbf{m}} \cdot \partial_{x} \hat{\mathbf{m}}+D(\hat{\mathbf{z}} \times \hat{\mathbf{x}}) \cdot\left(\hat{\mathbf{m}} \times \partial_{x} \hat{\mathbf{m}}\right)$, also gets minimized when $\tilde{\partial}_{x} \hat{\mathbf{m}}=0$. This is not a coincidence as this sum by definition should agree with the energy landscape of the Hamiltonian, which forces the value $D$ in Eq. (9).

One consequence of deriving the spin-orbit torque using the chiral derivative is that such a derivation shows that the spin-orbit torque is chiral when combined with the conventional spin torque just as the DM interaction is chiral when combined with the micromagnetic exchange interaction. For example, when $\mathbf{j}$ is along the $x$ direction, the total torque $\mathbf{T}_{\text {st }}$ in Eq. (11) vanishes even for finite $j$ if $\tilde{\partial}_{x} \hat{\mathbf{m}}=0$. As a side remark, the first and second terms in Eq. (11) are nothing but current-dependent corrections to the torques due to the total equilibrium energy density in Eq. (8) and the Gilbert damping, respectively. This identification is a straightforward generalization of a previously reported counterpart; when $\mathcal{H}_{\mathrm{R}}$ is absent, the adiabatic and nonadiabatic spin torques in Eq. (10) are the current-dependent corrections to the torques due to the micromagnetic exchange interaction [38] and the Gilbert damping 25].

The anomalously fast current-driven domain wall motion demonstrated in Ref. [9] raises the possibility that chirally ordered magnetic structures [ $[5,[6]$ such as topological Skyrmion lattices may be very efficiently controlled electrically. Such motion would be similar to the highly efficient electrically driven dynamics of a Skyrmion lattice in a system with bulk spin-orbit coupling such as the B20 structure 39]. Flexible deformation of the Skyrmion lattice is proposed [40] as an important contribution to the high efficiency of current-driven dynamics in B20 structures. We expect Skyrmion lattices in magnetic bilayers to behave similarly because both systems are similarly frustrated. The chiral derivative is noncommutative, $\tilde{\partial}_{x} \tilde{\partial}_{y} \hat{\mathbf{m}} \neq \tilde{\partial}_{y} \tilde{\partial}_{x} \hat{\mathbf{m}}$, so the energy landscape of the lattice structure is necessarily frustrated leading to 
the existence of many metastable structures with low excitation energies.

In a Skyrmion lattice, another linear effect of the interfacial spin-orbit coupling becomes important. Consider a Skyrmion lattice without interfacial spin-orbit coupling. The spatial variation of $\hat{\mathbf{m}}$ introduces a real space Berry phase [41], which can affect the electron transport through a Skyrmion lattice. It produces a fictitious magnetic field [42] $\mathbf{B}^{ \pm}=\mp(h / e) \hat{\mathbf{z}} b$, where $b=\left(\partial_{x} \hat{\mathbf{m}} \times \partial_{y} \hat{\mathbf{m}}\right) \cdot \hat{\mathbf{m}} / 4 \pi$ is nothing but the Skyrmion number density [41]. Here the upper and lower signs apply to majority (spin antiparallel to $\hat{\mathbf{m}}$ ) and minority (spin parallel to $\hat{\mathbf{m}}$ ) electrons, and thus this field is spin dependent. An experiment [6] on Fe/Ir bilayer reported the Skyrmion spacing of $1 \mathrm{~nm}$. For a Skyrmion density of $(1 \mathrm{~nm})^{-2}, \mathbf{B}^{ \pm}$becomes of the order of $10^{4} \mathrm{~T}$, which can significantly affect electron transport.

In the presence of interfacial spin-orbit coupling, the Berry-phase-derived field becomes chiral. Following the same procedure as above, one finds that $\mathbf{B}^{ \pm}$is now given by $\mp(h / e) \hat{\mathbf{z}} \tilde{b}$, where $\tilde{b}=\left(\tilde{\partial}_{x} \hat{\mathbf{m}} \times \tilde{\partial}_{y} \hat{\mathbf{m}}\right) \cdot \hat{\mathbf{m}} / 4 \pi=b+b_{\mathrm{R}}+$ $\mathcal{O}\left(\alpha_{\mathrm{R}}^{2}\right)$, where

$$
b_{\mathrm{R}}=k_{\mathrm{R}} \boldsymbol{\nabla} \cdot \hat{\mathbf{m}} / 4 \pi \text {. }
$$

We estimate the magnitude of $b_{\mathrm{R}}$ for the $\mathrm{Mn} / \mathrm{W}$ bilayer [5], for which left-handed cycloidal spiral with period $12 \mathrm{~nm}$ is reported. From the estimated value $D=$ $23.8 /(2 \pi) \mathrm{nm} \mathrm{meV}$ per $\mathrm{Mn}$ atom and $A=94.2 /(2 \pi)^{2}$ $\mathrm{nm}^{2}$ meV per Mn atom, we find $k_{\mathrm{R}}=0.794 \mathrm{~nm}^{-1}$ from Eq. (9), and $(h / e) b_{\mathrm{R}}$ becomes about $140 \mathrm{~T}$. Thus for the left-handed cycloidal spiral, for which the Skyrmion density $b=0$, the effective magnetic field is governed by this interfacial spin-orbit coupling contribution.

For completeness, we also discuss briefly the interfacial spin-orbit coupling contribution to the fictitious electric field $\mathbf{E}^{ \pm}$, which is spin dependent and arises when $\hat{\mathbf{m}}$ varies in time. Without $\mathcal{H}_{\mathrm{R}}$, it is known that $\mathbf{E}^{ \pm}=$ $\pm(h / 4 \pi e)\left(\mathbf{e}^{\text {adia }}+\mathbf{e}^{\text {non }}\right)$, where the so-called adiabatic contribution 42 44] is given by $\left(\mathbf{e}^{\text {adia }}\right)_{u}=\left(\partial_{t} \hat{\mathbf{m}} \times \partial_{u} \hat{\mathbf{m}}\right) \cdot \hat{\mathbf{m}}$ and the nonadiabatic contribution [45, 46] is given by $\left(\mathbf{e}^{\text {non }}\right)_{u}=\beta\left(\partial_{u} \hat{\mathbf{m}} \cdot \partial_{t} \hat{\mathbf{m}}\right)$. In the presence of $\mathcal{H}_{\mathrm{R}}$, corrections arise. Recently some of us [26] reported a correction term $\mathbf{e}_{\mathrm{R}}^{\text {adia }}$, and Ref. [47] reported another correction term $\mathbf{e}_{\mathrm{R}}^{\text {non }}$, which are given by

$$
\begin{aligned}
\left(\mathbf{e}_{\mathrm{R}}^{\text {adia }}\right)_{u} & =-k_{\mathrm{R}}(\hat{\mathbf{z}} \times \hat{\mathbf{u}}) \cdot \partial_{t} \hat{\mathbf{m}} \\
\left(\mathbf{e}_{\mathrm{R}}^{\text {non }}\right)_{u} & =\beta k_{\mathrm{R}}(\hat{\mathbf{z}} \times \hat{\mathbf{u}}) \cdot\left(\hat{\mathbf{m}} \times \partial_{t} \hat{\mathbf{m}}\right) .
\end{aligned}
$$

Here we point out that the previously reported corrections can be derived almost trivially using the chiral derivative since $\left(\mathbf{e}^{\text {adia }}+\mathbf{e}_{\mathrm{R}}^{\text {adia }}\right)_{u}=\left(\partial_{t} \hat{\mathbf{m}} \times \tilde{\partial}_{u} \hat{\mathbf{m}}\right) \cdot \hat{\mathbf{m}}$ and $\left(\mathbf{e}^{\text {non }}+\mathbf{e}_{\mathrm{R}}^{\text {non }}\right)_{u}=\beta\left(\tilde{\partial}_{u} \hat{\mathbf{m}} \cdot \partial_{t} \hat{\mathbf{m}}\right)$. This derivation also reveals the chiral nature of $\mathbf{e}_{\mathrm{R}}^{\text {adia }}$ and $\mathbf{e}_{\mathrm{R}}^{\text {non }}$. For the drift motion of chiral magnetic structures at $100 \mathrm{~m} / \mathrm{s}$, the parameter values of the $\mathrm{Mn} / \mathrm{W}$ bilayer [5] lead to the estimation that both $(h / 4 \pi e)\left(\mathbf{e}^{\text {adia }}\right)$ and $(h / 4 \pi e)\left(\mathbf{e}_{\mathrm{R}}^{\text {adia }}\right)$ are of the order of $10^{4} \mathrm{~V} / \mathrm{m}$, which should be easily detectable.

So far we focused on magnetic bilayers. But these results should also be relevant for the high-mobility $2 \mathrm{D}$ electron gas formed at the interface between two different insulating oxide materials. One example is the $\mathrm{LaAlO}_{3} / \mathrm{SrTiO}_{3}$ interface [48], which has broken structural inversion symmetry [49] and becomes magnetic [50] under proper conditions.

Last, we briefly discuss how features of real systems might affect our conclusions. Two differences in realistic band structures, are that the energy-momentum dispersion is not parabolic and that there are multiple energy bands [51]. Another difference is that magnetic bilayers are not strictly 2D systems, unlike systems such as $\mathrm{LaAlO}_{3} / \mathrm{SrTiO}_{3}$. To test the effects of more realistic band structures, in the Supplementary Material [30], we examine a tight-binding version of $\mathcal{H}$, which generates nonparabolic energy bands, and find that the relation (9) remains valid despite the nonparabolic dispersion. The two dimensionality is tested in a recent publication by some of us 52]. There, we perform a three-dimensional Boltzmann calculation to address the interfacial spinorbit coupling effect on the spin-orbit torque and obtain results, which are in qualitative agreement with those of the 2D Rashba model. On the basis of these observations, we expect that predictions of the simple Rashba model will survive at least qualitatively even in realistic situations and thus can serve as a good reference point for more quantitative future analysis.

To conclude, we examined effects of interfacial spinorbit coupling using the Rashba model. We found that all linear effects of the interfacial spin-orbit coupling can be derived by replacing spatial derivatives with chiral derivatives. This allows these effects to be understood in terms of chiral generalizations of effects in the absence of spin-orbit coupling. One important consequence is a relationship between the DM interaction and the spin-orbit torque, such that measuring one should give a strong indication of the other.

H.W. L. wishes to thank Ki-Seok Kim, F. Freimuth, S. Blügel, T. Silva, and P. Haney for critical comments and useful discussions. Financial support was provided by NRF Grants No. 2013R1A2A2A05006237 and No. 20110030046 (H.W. L.) and No. 2013R1A2A2A01013188 (K. J. L.).

* Electronic address: hwl@postech.ac.kr

[1] I. M. Miron, G. Gaudin, S. Auffret, B. Rodmacq, A. Schuhl, S. Pizzini, J. Vogel, and P. Gambardella, Nat. Mater. 9, 230 (2010).

[2] I. M. Miron, T. Moore, H. Szambolics, L. D. BudgaPrejbeanu, S. Auffret, B. Rodmacq, S. Pizzini, J. Vogel, M. Bonfim, A. Schuhl, and G. Gaudin, et al., Nat. Mater. 
10, 419 (2011).

[3] I. M. Miron, K. Garello, G. Gaudin, P.-J. Zermatten, M. V. Costache, S. Auffret, S. Bandiera, B. Rodmacq, A. Schuhl, and G. Gambardella, et al., Nature (London) 476, 189 (2011).

[4] L. Liu, C.-F. Pai, Y. Li, H. W. Tseng, D. C. Ralph, and R. A. Buhrman, Science 4, 555 (2012).

[5] M. Bode, M. Heide, K. von Bergmann, P. Ferriani, S. Heinze, G. Bihlmayer, A. Kubetzka, O. Pietzsch, S. Blügel, and R. Wiesendanger, Nature (London) 447, 190 (2007).

[6] S. Heinze, K. von Bergmann, M. Menzel, J. Brede, A. Kubetzka, R. Wiesendanger, G. Bihlmayer, and S. Blügel, Nat. Phys. 7, 713 (2011).

[7] A. Thiaville, S. Rohart, É Jué, V. Cros, and A. Fert, Europhys. Lett. 100, 57002 (2012).

[8] S. Emori, U. Bauer, S.-M. Ahn, E. Martinez, and G. S. Beach, Nat. Mater. 12, 611 (2013)

[9] K.-S. Ryu, L. Thomas, S.-H. Yang, and S. Parkin, Nat. Nanotech. 8, 527 (2013).

[10] I. E. Dzyaloshinskii, Sov. Phys. JETP 5, 1259 (1957).

[11] T. Moriya, Phys. Rev. 120, 91 (1960).

[12] A. Fert, Mater. Sci. Forum 59 and 60, 439 (1990)

[13] K. Obata and G. Tatara, Phys. Rev. B 77, 214429 (2008).

[14] A. Matos-Abiague and R. L. Rodriguez-Suarez, Phys. Rev. B 80, 094424 (2009).

[15] X. Wang and A. Manchon, Phys. Rev. Lett. 108, 117201 (2012).

[16] K.-W. Kim, S. M. Seo, J. Ryu, K.-J. Lee, and H.-W. Lee, Phys. Rev. B 85, 180404 (2012).

[17] D. A. Pesin and A. H. MacDonald, Phys. Rev. B 86, 014416 (2012).

[18] E. van der Bijl and R. A. Duine, Phys. Rev. B 86, 094406 (2012).

[19] L. Liu, O. J. Lee, T. J. Gudmundsen, D. C. Ralph, and R. A. Buhrman, Phys. Rev. Lett. 109, 096602 (2012).

[20] J. Kim, J. Sinha, M. Hayashi, M. Yamanouchi, S. Fukami, T. Suzuki, S. Mitani, and H. Ohno, Nat. Mater. 12, 240 (2013).

[21] P. P. J. Haazen et al., Nat. Mater. 12, 299 (2013).

$[22]$ F. Freimuth, S. Blügel, and Y. Mokrousov, arXiv:1305.4873.

[23] H. Kohno, G. Tatara, and J. Shibata, J. Phys. Soc. Jpn. 75, 113706 (2006).

[24] R. A. Duine, A. S. Núñez, J. Sinova, and A. H. MacDonald, Phys. Rev. B 75, 214420 (2007).

[25] I. Garate and A. MacDonald, Phys. Rev. B 79, 064404 (2009).

[26] K.-W. Kim, J.-H. Moon, K.-J. Lee, and H.-W. Lee, Phys. Rev. Lett. 108, 217202 (2012).

[27] I. Garate, K. Gilmore, M. D. Stiles, and A. H. MacDon- ald, Phys. Rev. B 79, 104416 (2009).

[28] I. L. Aleiner and V. I. Fal'ko, Phys. Rev. Lett. 87, 256801 (2001).

[29] M. Valin-Rodriguez, Phys. Rev. Lett. 107, 266801 (2011).

[30] See the Supplemental material at Phys. Rev. Lett. for details.

[31] H. Imamura, P. Bruno, and Y. Utsumi, Phys. Rev. B 69, 121303 (2004).

[32] S. Lounis, A. Bringer, and S. Blügel, Phys. Rev. Lett. 108, 207202 (2012).

[33] D. C. Ralph and M. D. Stiles, J. Magn. Magn. Mater. 320, 1190 (2008).

[34] G. Tatara and H. Kohno, Phys. Rev. Lett. 92, 086601 (2004).

[35] S. Zhang and Z. Li, Phys. Rev. Lett. 93, 127204 (2004).

[36] A. Thiaville, Y. Nakatani, J. Miltat, and Y. Suzuki, Europhys. Lett. 69, 990 (2005).

[37] S. Datta and B. Das, Appl. Phys. Lett. 56, 665, (1990).

[38] J. Q. Xiao, A. Zangwill, and M. D. Stiles, Phys. Rev. B 73, 054428 (2006).

[39] F. Jonietz et al. Science 330, 1648 (2010).

[40] J. Iwasaki, M. Mochizuki, and N. Nagaosa, Nat. Commun. 4, 1463 (2013).

[41] A. Neubauer et al. Phys. Rev. Lett. 102, 186602 (2009).

[42] G. E. Volovik, J. Phys. C 20, L83 (1987).

[43] S. E. Barnes and S. Maekawa, Phys. Rev. Lett. 98, 246601 (2007).

[44] S. A. Yang, G. S. D. Beach, C. Knutson, D. Xiao, Z. Zhang, M. Tsoi, Q. Niu, A. H. MacDonald, and J. L. Erskine, Phys. Rev. B 82, 054410 (2010).

[45] R. A. Duine, Phys. Rev. B 77, 014409 (2008).

[46] Y. Tserkovnyak and M. Mecklenburg, Phys. Rev. B 77, 134407 (2008).

[47] G. Tatara, N. Nakabayashi, and K.-J. Lee, Phys. Rev. B 87, 054403 (2013).

[48] A. Ohtomo and H. Y. Hwang, Nature (London) 427, 423 (2004).

[49] A. D. Caviglia, M. Gabay, S. Gariglio, N. Reyren, C. Cancellieri, and J.-M. Triscone, Phys. Rev. Lett. 104, 126803 (2010).

[50] A. Brinkman, H. Huijben, M. van Zalk, J. Huijben, U. Zeitler, J. C. Maan, W. G. ven der Wiel, G. Rijnders, D. H. A. Blank, and H. Hilgenkamp, Nat. Mater. 6, 493 (2007).

[51] J.-H. Park, C. H. Kim, H.-W. Lee, and J.-H. Han, Phys. Rev. B 87, 041301 (2013).

[52] P. M. Haney, H.-W. Lee, K.-J. Lee, A. Manchon, and M. D. Stiles, Phys. Rev. B 87, 174411 (2013). 